\section{Betablocker fördert Heilung von Brandwunden}

\author{
US-Chirurgen haben untersucht, ob Propranolol auch die Wundheilung und \\ die perioperative Hämodynamik bei schweren Brandverletzungen positiv \\ beeinflussen kann - mit vielversprechenden Ergebnissen.
}

$B^{e}$ ei Patienten mit schweren Brandveretzungen sind oft mehrere Eingriffe erforderlich, um die motorische Funktionalität wieder herzustellen und kosmetisch zufriedenstellende Ergebnisse zu erzielen. Häufig stehen solche Prozeduren gerade dann an, wenn das intraoperative Blutungsrisiko am höchsten ist, nämlich zwei bis 16 Tage nach der Verletzung. So sucht man nach pharmazeutischen Optionen, den Blutverlust während der Operationen so gering wie möglich zu halten.

Es hat sich gezeigt, dass Propranolol durch seine Eigenschaft als Vasokonstriktor die Gefahr peripherer Blutungen nach Brandverletzungen verringert und den Gefäßwiderstand erhöht. Chirurgen aus den USA haben nun untersucht, ob die Veränderungen der kardiovaskulären Situation durch den nicht selektiven Betablocker auch die Wundheilung und die perioperative Hämodynamik bei Erwachsenen mit schweren Brandverletzungen positiv beeinflussen können.
Hierzu wurden 69 Probanden mit Verbrennungen von mindestens $30 \%$ der Körperoberfläche in eine prospektive Studie aufgenommen. 34 Probanden der Kontrollgruppe wurden nach dem Standardprogramm behandelt. Die übrigen 35 Verbrennungsopfer erhielten innerhalb von 48 Stunden zusätzlich täglich $3,3 \pm 3,0 \mathrm{mg} / \mathrm{kg}$ Propranolol über durchschnittlich 40 Tage. Damit konnte während der ersten 30 Kliniktage die Herzfrequenz der Patienten signifikant um etwa $20 \%$ gedrosselt werden. Von der Exzision der Brandwunden bis zur Entlassung bekamen die Patienten mehrere Hauttransplantationen. Sobald die Spenderstelle verheilt war, erfolgte der nächste Eingriff. So ergab sich aus den Zeiträumen zwischen den einzelnen Transplantationen ein Maß für die Schnelligkeit der Wundheilung. Das Ausmaß des perioperativen Blutverlusts wurde durch den prä- und postoperativen Hämatokritwert bestimmt.
Die Patienten der Kontroll- und der Propranolol-Gruppe benötigten im Schnitt vier Hauttransplantationen. Unterschiedlich war allerdings die Länge der Pausen zwischen den einzelnen Eingriffen. Bei den Probanden der Propranololgruppe vergingen durchschnittlich $10 \pm 5$ Tage, bei Patienten der Kontrollgruppe hingegen $17 \pm 12$ Tage. Dieser Unterschied spricht für eine schnellere Heilung unter einer Therapie mit Propranolol. Die Patienten der Propranolol-Gruppe erhielten signifikant größere Hauttransplantate als die der Kontrollgruppe. Dennoch war der Blutverlust in beiden Gruppen vergleichbar, sodass zur Aufrechterhaltung des perioperativen Hämatokrits jeweils ähnlich viele Erythrozytenkonzentrate benötigt wurden.

Fazit: Die Gabe von Propranolol drosselt bei Patienten mit schweren Brandwunden den Blutverlust während der ersten Hauttransplantationen. Zudem heilen Wunden unter Einfluss des Betablockers schneller.

Dr. Christine Starostzik

Ali A et al. Propranolol attenuates hemorrhage and accelerates wound healing in severely burned adults. Crit Care 2015; 19: 217

\title{
Vorsicht beim Wechsel von Autoinjektoren!
}

Viele Patienten wenden ihre Adrenalin-Autoinjektoren (AAl) falsch an. Bislang war aber unklar, ob das an mangelhaften Anleitungen oder am Geräteaufbau liegt. Einer Gerätetausch-Studie zufolge könnte Letzteres die Ursache sein.

n einer prospektiven, randomisierten Studie wurden zunächst Eltern von anaphylaxiegefährdeten Kindern kontaktiert, die erstmalig einen Adrenalin-Autoinjektor (AAI) - entweder Anapen ${ }^{\circledast}$ oder EpiPen ${ }^{ø}$ - erhielten. Die 158 Eltern wurden gemäß Herstellerangaben geschult und zeigten nach Abschluss der Schulung, dass sie imstande waren, die Injektion korrekt durchzuführen. Nach sechs Wochen und einem Jahr wurden die Fertigkeiten der Eltern in einer simulierten Anaphylaxie unter Stressbedingungen getestet. Zudem bekamen sie nach einem Jahr randomisiert einen neuen AAI (EpiPen ${ }^{\varpi}$, Anapen ${ }^{\oplus}$, Jext ${ }^{\oplus}$, ein neu- es EpiPen ${ }^{\circledast}$-Modell oder Auvi-Q ${ }^{\mathrm{TM}}$, einen „sprechenden“ AAI, der bislang nur in den USA erhältlich ist). Diesmal gab es keinerlei spezifische Schulungen.

Nach sechs Wochen verwendeten $42 \%$ ihren Anapen ${ }^{\varpi}$ und $43 \%$ ihren EpiPen ${ }^{\oplus}$ korrekt, auch nach einem Jahr waren die Erfolgsraten mit $55 \%$ und 59\% vergleichbar - und jeweils besorgniserregend niedrig. Hauptgrund für Versagen war, dass die Eltern daran scheiterten, den Sicherheitsverschluss zu öffnen, gefolgt von zu kurzer Anwendungsdauer $(<5 \mathrm{~s})$.

Mit welchem AAI die Teilnehmer in der ersten Studienphase geschult worden waren, hatte keinen Einfluss auf die Er- folgsraten nach dem Gerätetausch. Entscheidend war vielmehr, welcher AAI in der zweiten Phase verwendet wurde. Mit dem Auvi-Q ${ }^{\mathrm{TM}}$ konnten $93 \%$ der Eltern erfolgreich Adrenalin injizieren im Vergleich zu $49 \%$ mit allen anderen AAI. Besonders schlecht schnitten Eltern ab, die von einem AAI mit zwei Sicherheitskappen (Anapen ${ }^{\circ}$ ) auf einen mit einer Kappe (alle anderen) wechselten und umgekehrt. Die Autoren empfehlen Allergologen, diesen Umstand zu berücksichtigen, wenn ihre Patienten den AAI wechseln.

Fazit: Die Beschaffenheit eines AAI scheint entscheidend zu beeinflussen, ob das jeweilige Gerät korrekt angewendet wird. Audiogestützte AAI erzielen die besten Erfolgsraten.

Sebastian Lux

Umasunthar et al. Patients' ability to treat anaphylaxis using adrenaline autoinjectors: a randomized controlled trial. Allergy 2015; 70 : 855-63 